PROCEEDINGS OF THE

AMERICAN MATHEMATICAL SOCIETY

Volume 126, Number 12, December 1998, Pages 3539-3544

S 0002-9939(98)05082-5

\title{
A NECESSARY AND SUFFICIENT CONDITION FOR UPPER HEMICONTINUOUS SET-VALUED MAPPINGS WITHOUT COMPACT-VALUES BEING UPPER DEMICONTINUOUS
}

\author{
GAN-SHANG YANG AND GEORGE XIAN-ZHI YUAN
}

(Communicated by David R. Larson)

\begin{abstract}
The purpose of this article is to give a characterization of an upper hemicontinuous mapping with non-empty convex values being upper demicontinuous, i.e., we show that an upper hemicontinuous set-valued mapping with non-empty convex values (not necessarily compact-valued) is upper demicontinuous if and only if the set-valued mapping has no interior asymptotic plane.
\end{abstract}

\section{INTRODUCTION}

When we study nonlinear set-value problems such as existence of fixed points for set-valued mappings, the existence of solutions for differential inclusions, and others, we often face the problem of dealing with different kinds of continuity for setvalued mappings. In the case of the single-valued mapping, the continuity defined by neighborhood is equivalent with that definition by using language of sequences or nets. However, in the case of strict set-valued mappings, these two definitions are no longer equivalent. As a result, a few concepts about continuity of set-valued mappings, for example, upper semicontinuity, upper demicontinuity, and upper hemicontinuity, have been introduced and often used in the study of non-linear setvalued analysis (e.g., see Aubin and Ekeland [1], Aubin and Frankowska [2], Fan [3], Klein and Thompson [4] and related references therein). Thus, it is necessary to illustrate the distinction of intrinsic quality among these different continuities. In order to bring readers to the problem we wish to study, we first recall three concepts of continuity for set-valued mappings.

Let $X$ and $Y$ be two topological spaces and $F: X \rightarrow 2^{Y}$ a set-valued mapping. As usual, we say that $F$ is upper semicontinuous (in short, USC) on $X$ if for each $x \in X$ and for each open set $G$ in $Y$ with $F(x) \subset G$, there exists an open neighborhood $N_{x}$ of $x$ in $X$ such that $F(z) \subset G$ for all $z \in N_{x}$. Let $E^{*}$ be the dual space (i.e., the vector space of all continuous linear functionals) of a real Hausdorff topological vector space $E$. According to Fan [3], a set-valued mapping $F: X \rightarrow 2^{E}$ is said to be upper demicontinuous (in short, UDC) on $X$ if for each $x \in X$ and any

Received by the editors February 26, 1997.

1991 Mathematics Subject Classification. Primary 47H04, 54C60.

Key words and phrases. Upper semicontinuous, upper hemicontinuous, upper demicontinuous, asymptotic plane, interior asymptotic plane.

This project was supported in part by the Australian Research Council.

(C)1998 American Mathematical Society 
open half-space (or, say, hyper-plane) $H$ in $E$ containing $F(x)$, i.e., $F(x) \subset H$, there exists an open neighborhood $N_{x}$ of $x$ in $X$ such that $F(z) \subset H$ for all $z \in N_{x}$. We recall that an open half-plane $H$ in $E$ is a set of the form $H:=\{x \in E: \phi(x)<\lambda\}$ for some $\phi \in E^{*}$ and $\lambda \in R$. By following Aubin and Ekeland [1, p.122], a setvalued mapping $F: X \rightarrow 2^{E}$ is said to be upper hemicontinuous on $X$ (in short, UHC) if for each $\phi \in E^{*}$ and for each $\lambda \in R$, the set $\left\{x \in X: \sup _{u \in F(x)} \phi(u)<\lambda\right\}$ is open in $X$.

From the definitions, it is obvious that each upper semicontinuous set-valued mapping is upper demicontinuous and each upper demicontinuous set-valued mapping is upper hemicontinuous, but the converse is not true in general. However, if a set-valued mapping is compact-valued, the upper hemicontinuity implies the upper demicontinuity. In this case, the concepts of upper demicontinuity and upper hemicontinuity coincide. Furthermore, when the image space of a set-valued mapping with compact-value is compact, all three definitions above are equivalent, e.g., see Proposition 2 of Shih and Tan [5, p.238] or some other discussion from books of Aubin and Ekeland [1], Aubin and Frankowska [2] and Klein and Thompson [4]. But, under what kind of conditions is the upper hemicontinuity equivalent to the upper demicontinuity without the assumption of compactness for set-valued mappings? To the best of our knowledge, there is no result which is available so far in this direction. It is our aim to study this question. In this paper we give a characterization of an upper hemicontinuous mapping with non-empty convex values (not necessarily compact-valued) being upper demicontinuous, i.e., we show that an upper hemicontinuous set-valued mapping with non-empty convex values is upper demicontinuous if and only if the mapping $F$ has no interior asymptotic plane. As applications, corresponding results in the literature have been improved or derived in a shorter form.

\section{The DEFINITION OF ASYMPtotic Planes}

Throughout this article, let $X$ be a Hausdorff topological space and $E$ a real Hausdorff locally convex topological linear space. For the sake of convenience, we first introduce the definition of asymptotic plane which could give us the characterization of upper hemicontinuity being upper demicontinuous for set-valued mappings with no compact values.

Definition 1. Let $E^{*}$ be the dual space of a Hausdorff locally convex topological linear space $E$ and $A$ a non-empty subset of $E$. For each given $\phi \in E^{*}$ and $\lambda \in R$, the set $M_{\phi, \lambda}=\{x \in E: \phi(x)=\lambda\}$ is said to be an asymptotic plane of $A$ respective to $(\phi, \lambda) \in E^{*} \times R$ (in short, $M_{\phi, \lambda}$ is said to be an asymptotic plane of $A$ if there is no confusion in the content) if $M_{\phi, \lambda} \cap A=\emptyset$ and there exists a positive number $\delta \in R$ such that $M_{\phi, \mu} \cap A \neq \emptyset$ for all $\mu \in(\lambda-\delta, \lambda)$.

Remark 1. By Definition 1 above, we know that all compact sets and weakly convex compact sets in the locally topological vector space $E$ have no asymptotic planes (see the proofs of Corollaries 1 and 2 below for details).

Definition 2. Let $X$ be a Hausdorff topological space, $E$ a Hausdorff locally convex topological linear space, and $F: X \rightarrow 2^{E} \backslash\{\emptyset\}$ a set-valued mapping with non-empty values. For any given point $x_{0} \in X$, the asymptotic plane $M_{\phi, \lambda}$ of $F\left(x_{0}\right)$ is said to be a boundary asymptotic plane of $F$ at $x_{0}$, if there exists an open 
neighborhood $N\left(x_{0}\right)$ of $x_{0}$ such that

$$
F(x) \subset H_{\phi, \lambda}=\{y \in E: \phi(y)<\lambda\}
$$

for all $x \in N\left(x_{0}\right)$. The asymptotic plane $M_{\phi, \lambda}$ of $F\left(x_{0}\right)$ is said to be an interior asymptotic plane of $F$ at $x_{0}$, if for any neighborhood $N\left(x_{0}\right)$ of $x_{0}$, there exists $x \in N\left(x_{0}\right)$ such that

$$
F(x) \not \subset H_{\phi, \lambda}:=\{y \in E: \phi(y)<\lambda\} .
$$

If for all $x \in X$, the set $F(x)$ has no interior asymptotic plane, then the mapping $F$ is said to have no interior asymptotic plane.

Remark 2. From the definition, it follows that each asymptotic plane of a given set $A$ in $E$ is either a boundary or interior asymptotic plane. Now we have the following characterization for a set-valued upper hemicontinuous mapping being upper demicontinuous.

Theorem 1. Let $X$ be a Hausdorff topological space and $E$ a Hausdorff locally convex topological linear space. Suppose $F: X \rightarrow 2^{E}$ is an upper hemicontinuous set-valued mapping with non-empty convex values (not necessarily compact-valued). Then $F$ is upper demicontinuous if and only if the mapping $F$ has no interior asymptotic plane on $X$.

Proof. Necessity: Suppose the conclusion is false. Then there exist $x_{0} \in X$ and an asymptotic plane $M_{\phi, \lambda}$ at $x_{0}$ such that $M_{\phi, \lambda}$ is an interior asymptotic plane of $F\left(x_{0}\right)$ for some $\phi \in E^{*}$ and $\lambda \in R$. By Definition 1 , there exists some $\delta>0$, such that for any $\mu \in(\lambda-\delta, \lambda)$, we have that $H_{\phi, \lambda} \cap F\left(x_{0}\right) \supset M_{\phi, \mu} \cap F\left(x_{0}\right) \neq \emptyset$. Next we want to show that $F\left(x_{0}\right) \subset H_{\phi, \lambda}$. In fact, if there exists $y \in F\left(x_{0}\right)$ such that $y \notin H_{\phi, \lambda}$, it follows that $\phi(y) \geq \lambda$. Taking $y_{0} \in H_{\phi, \lambda} \cap F\left(x_{0}\right)$, we have that $\phi\left(y_{0}\right)<\lambda$. Let

$$
y_{1}:=\frac{\phi(y)-\lambda}{\phi(y)-\phi\left(y_{0}\right)} y_{0}+\frac{\lambda-\phi\left(y_{0}\right)}{\phi(y)-\phi\left(y_{0}\right)} y .
$$

We know that $y_{1} \in F\left(x_{0}\right)$ and $\phi\left(y_{1}\right)=\lambda$. Thus $M_{\phi, \lambda} \cap F\left(x_{0}\right) \neq \emptyset$. This contradicts our assumption that $M_{\phi, \lambda}$ is an asymptotic plane of $F\left(x_{0}\right)$. Therefore, we must have that $F\left(x_{0}\right) \subset H_{\phi, \lambda}$. Secondly, by the definition of interior asymptotic planes, for neighborhood $N\left(x_{0}\right)$ of $x_{0}$, there exists $x \in N\left(x_{0}\right)$ such that $F(x) \not \subset M_{\phi, \lambda}$. This contradicts our assumption that $F$ is demicontinuous at $x_{0} \in X$. Hence, $F$ has no interior asymptotic plane at $x_{0}$.

Sufficiency: Suppose that $F$ is not demicontinuous on $X$. Then there exists $x_{0} \in X$ and an open half-space $H_{\phi, \lambda}=\{y \in E: \phi(y)<\lambda\}$ with $F\left(x_{0}\right) \subset H_{\phi, \lambda}$ for some $\phi \in E^{*}$ and $\lambda \in R$, such that for any neighborhood $N\left(x_{0}\right)$ of $x_{0}$, there exists $x \in N\left(x_{0}\right)$ such that $F(x) \not \subset H_{\phi, \lambda}$. Therefore, for any neighborhood $N\left(x_{0}\right)$ of $x_{0}$, there exist $x_{N\left(x_{0}\right)} \in N\left(x_{0}\right)$ and $y_{N\left(x_{0}\right)} \in F\left(x_{N\left(x_{0}\right)}\right)$ such that $\phi\left(y_{N\left(x_{0}\right)}\right) \geq \lambda$. In what follows, we want to show that $M_{\phi, \lambda}$ is not a boundary asymptotic plane of $F\left(x_{0}\right)$. In order to do so, it suffices to show that $M_{\phi, \lambda}$ is an asymptotic plane of $F\left(x_{0}\right)$. Now let the net $\left\{x_{v}\right\}_{v \in \mathcal{V}}$ be a direct family of the set $\left\{N_{V}\left(x_{0}\right)\right\}_{v \in \mathcal{V}}$ with $\lim _{v \in \mathcal{V}} x_{v}:=x_{0}$, where $\left\{N_{v}\left(x_{0}\right)\right\}_{v \in \mathcal{V}}$ is the family of all neighborhoods $N\left(x_{0}\right)$ at the point $x_{0}$. As we just discussed above, for each neighborhood $N_{v}\left(x_{0}\right)$ of $x_{0}$, there exist $x_{v} \in N_{v}\left(x_{0}\right)$ and $y_{v} \in F\left(x_{v}\right)$ such that

$$
\operatorname{Sup}_{u \in F\left(x_{v}\right)} \phi(u) \geq \phi\left(y_{v}\right) \geq \lambda .
$$


By the hemicontinuity of $F$, we do have that $\operatorname{Sup}_{u \in F\left(x_{0}\right)}(u) \geq \varlimsup_{\lim _{v}} \sup _{u \in F\left(x_{v}\right)} \phi(u)$ $\geq \lambda$. Note that $F\left(x_{0}\right) \subset H_{\phi, \lambda}$; it follows that $\sup _{u \in F\left(x_{0}\right)} \phi(u)=\lambda$. Now take $y_{0} \in F\left(x_{0}\right)$; it follows that $\phi\left(y_{0}\right)<\lambda$ as $F\left(x_{0}\right) \subset H_{\phi, \lambda}$. Let $\delta=\lambda-\phi\left(y_{0}\right)$. We then have that $\delta>0$ and $(\lambda-\delta, \lambda)=\left(\phi\left(y_{0}\right), \lambda\right)$. For any $\mu \in(\lambda-\delta, \lambda)$ and $\sigma \in(\mu, \lambda)$, as $\operatorname{Sup}_{u \in F\left(x_{0}\right)} \phi(u)=\lambda$, there exists $y_{1} \in F\left(x_{0}\right)$ such that $\phi\left(y_{1}\right)>\sigma$. This implies that $\mu \in\left(\phi\left(y_{0}\right), \phi\left(y_{1}\right)\right)$. By the convexity of $F\left(x_{0}\right)$, we know that $F\left(x_{0}\right)$ is a path connected set. By the intermediate-value theorem, there exists $\hat{x} \in F\left(x_{0}\right)$ such that $\phi(\hat{x})=\mu$. It follows that $M_{\phi, \mu} \cap F\left(x_{0}\right) \neq \emptyset$ for all $\mu \in(\lambda-\delta, \lambda)$, i.e., $M_{\phi, \lambda}$ is an asymptotic plane of $F\left(x_{0}\right)$. Therefore the set $M_{\phi, \lambda}$ is not a boundary asymptotic plane of $F$ at $x_{0}$ and thus $M_{\phi, \lambda}$ is an interior asymptotic plane of $F$ at $x_{0}$. This contradicts our assumption that $F$ has no any interior asymptotic plane on $X$ and thus the proof is completed.

Example 1. Let $E:=R^{2}, X:=[0,1]$ and define a set-valued mapping from $X$ to $E$ by $F(x)=\{(u, v): u>x, v \geq 1 /(u-x)\}$ for each $x \in[0,1]$. Then $F$ : $X \rightarrow 2^{E}$ is a set-valued mapping with non-empty and convex values. For any given $\phi \in E^{*}$, without loss of generality, we might assume that the functional $\phi$ has the form $\phi(u, v):=a u+b v$, where both $a$ and $b$ are two constants determined by the functional $\phi$. If either $a$ or $b$ is positive, it is clear that $\sup _{(u, v) \in F(x)} \phi(u, v)=+\infty$. In the case both $a$ and $b$ are negative, we can also check that $\sup _{(u, v) \in F(x)} \phi(u, v)=$ $a x-2 \sqrt{a b}$. Thus the function $x \mapsto \operatorname{Sup}_{(u, v) \in F(x)} \phi(u, v)$ is continuous, which means that the set-valued mapping $F$ is upper hemicontinuous. However, for any $x \in X$, let $u:=x$. Then $u$ is an interior asymptotic plane of $F(x)$. By applying Theorem $1, F$ is not upper demicontinuous.

Corollary 1. Let $X$ be a Hausdorff topological space and $E$ a Hausdorff locally convex topological vector space. If the set-valued mapping $F: X \rightarrow 2^{E} \backslash\{\emptyset\}$ is upper hemicontinuous with non-empty and compact convex values, then $F$ is upper demicontinuous.

Proof. We first show that each compact set $A$ of $E$ has no asymptotic plane as pointed out in the previous Remark 1. Indeed, if $A$ has an asymptotic plane $M_{\phi, \lambda}$, where $\phi \in E^{*}$ and $\lambda \in R$, by Definition 1, for any $u \in A$, we have that $\phi(u) \neq \lambda$ and there exists $\delta>0$ such that for any $\mu \in(\lambda-\delta, \lambda)$, there is $\hat{x} \in A$ such that $\phi(\hat{x})=\mu$. Now define $B:=\{u \in A: \phi(u) \leq \lambda\}$. Then $B$ is also a compact subset of $E$. Therefore, there exists $u_{0} \in B$ such that $\phi\left(u_{0}\right)=\sup _{u \in B} \phi(u)$. By the arbitrariness of $\mu \in(\lambda-\delta, \lambda)$, it follows that $\phi\left(u_{0}\right)=\lambda$. Note that $M_{\phi, \lambda}$ is an asymptotic plane of $A$, and by the definition of the set $B$, it follows that for each $u \in B, \phi(u)<\lambda$. Therefore $\phi\left(u_{0}\right)<\lambda$. This is impossible as we just showed that $\phi\left(u_{0}\right)=\lambda$. Thus, the compact set $A$ in $E$ has no asymptotic planes. By Theorem $1, F$ is upper demicontinuous and the proof is completed.

As another application of Theorem 1, we have the following result which says that upper hemicontinuous mappings with non-empty convex and weakly compact values are also upper demicontinuous.

Corollary 2. Let $X$ be a Hausdorff topological space and $E$ a Hausdorff locally convex topological vector space. If the set-valued mapping $F: X \rightarrow 2^{E} \backslash\{\emptyset\}$ is hemicontinuous with non-empty weakly compact convex values, then $F$ is demicontinuous. 
Proof. If there exists $x \in X$ such that $F(x)$ has an asymptotic plane $M_{\phi, \lambda}$, then for any $u \in F(x)$, we have that $\phi(u) \neq \lambda$ and secondly, there exists $\delta>0$ such that for each $\mu \in(\lambda-\delta, \lambda)$, there is $u \in F(x)$ such that $\phi(u)=\mu$. Now define a set

$$
B:=\{u \in F(x): \phi(u) \leq \lambda\} .
$$

Then for any $u \in B$, it follows that $\phi(u)<\lambda$. Let $\left\{\mu_{n}\right\}_{n \in N}$ be a sequence of the set $(\lambda-\delta, \lambda)$ such that $\lim _{n \rightarrow \infty} \mu_{n}=\lambda$. Then by the definition of the set $B$ and our discussion above, there exists $\left\{u_{n}\right\} \subset B$ such that $\phi\left(u_{n}\right)=\mu_{n}$. As each weakly compact set in the Hausdorff locally convex topological vector space $E$ is weakly closed, $B$ is also convex weakly compact, and thus there exists a directed sub-family $\left\{u_{v}\right\}_{v \in D} \subset\left\{u_{n}\right\}$ and a point $u_{0} \in B$ such that for any $\phi \in E^{*}$, we have that $\lim _{v} \phi\left(u_{v}\right)=\phi\left(u_{0}\right)$. Therefore,

$$
\phi\left(u_{0}\right)=\lim _{v} \phi\left(u_{v}\right)=\lim _{v} \mu_{v}=\lambda .
$$

This contradicts that for each $u \in B, \phi(u)<\lambda$ as shown above. Therefore $F$ has no asymptotic planes. By Theorem $1, F$ is demicontinuous and the proof is completed.

Corollary 3. Let $X$ be a Hausdorff topological space and $E$ a reflexive Banach space. If the set-valued mapping $F: X \rightarrow 2^{E} \backslash\{\emptyset\}$ is hemicontinuous with nonempty bounded and closed convex set, then $F$ is demicontinuous.

Proof. Indeed, this is a special case of Corollary 2. But here we can also give a short proof by using Theorem 1. If there exists $x \in X$ such that $F(x)$ has an asymptotic plane $M_{\phi, \lambda}$, then on one hand, we know that the sets $F(x)$ and $M_{\phi, \lambda}$ cannot be strongly separated by any real hyper-plane; on the other hand, note that $E$ is a reflexive Banach space, $F(x)$ is bounded closed convex and so is $M_{\phi, \lambda}$, then the sets $F(x)$ and $M_{\phi, \lambda}$ can be strongly separated by a real hyper-plane. This is impossible. Finally the conclusion is derived by Theorem 1 .

Remark 3. Finally we would like to note that both Corollaries 1 and 2 improve corresponding results of Proposition 2 of Shih and Tan [5]. Also all results still hold if the locally topological vector space $E$ is replaced by a topological vector space (not necessarily having locally convex structure) with many sufficient continuous linear functionals.

\section{ACKNOWLEDGEMENT}

Both authors would like to express their sincere thanks to Professor David R. Larson and the anonymous referee for the careful reading and useful suggestions which led to the present version of this paper.

\section{REFERENCES}

1. J. P. Aubin and I. Ekeland, Applied Nonlinear Analysis, Wiley-Interscience, 1984. MR 87a:58002

2. J. P. Aubin and H. Frankowska, Set-valued Analysis, Birkhäuser, Boston, 1990. MR 91d:49001

3. K. Fan, A minimax inequality and applications, Inequalities III, Proceedings of Third Symposium on Inequalities (O. Shisha, ed.), Academic Press, New York, 1972, pp. 103-113. MR 49:5779

4. E. Klein and A. C. Thompson, Theory of Correspondences: including applications to mathematical economics, John Wiley and Sons, 1984. MR 86a:90012 
5. M. H. Shih and K. K. Tan, Covering theorems of convex sets related to fixed point theorems, Nonlinear and Convex Analysis-Proceedings in Honor of Ky Fan (B. L. Lin and S. Simons, eds.), Marcel Dekker, Inc., 1987, pp. 235-244. MR 88g:46019

Department of Mathematics, Yunnan National Institute, Kunming, China 650031

Department of Mathematics, The University of Queensland, Brisbane, Queensland, Australia 4072

E-mail address: xzy@maths.uq.edu.au 